\title{
Noticias de prensa aplicadas a la enseñanza del derecho de familia y sucesiones
}

\author{
Sofía de Salas Murillo y M$^{\mathrm{a}}$ Victoria Mayor del Hoyo ${ }^{\mathrm{b}}$

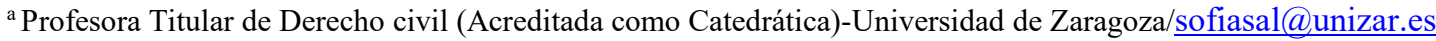 \\ b Profesora Titular de Derecho civil-Universidad de Zaragoza/mvmayor@unizar.es
}

\section{\$EWWFW}

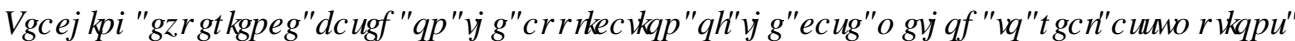

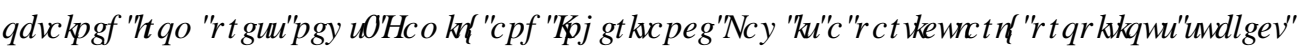

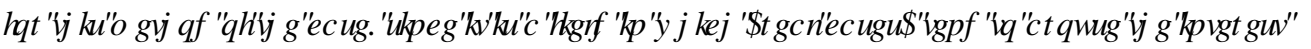

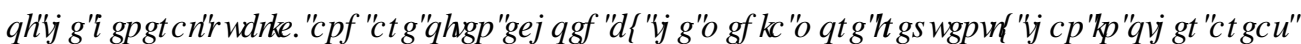

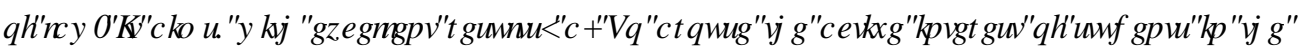

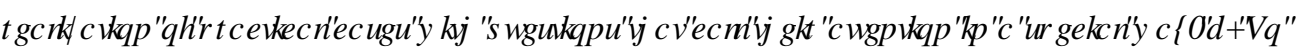

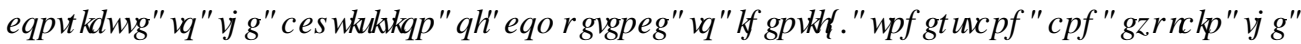

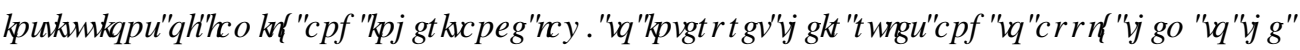

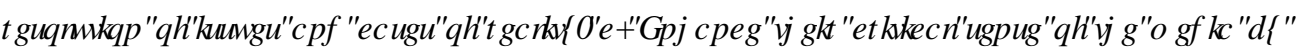

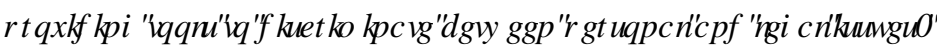

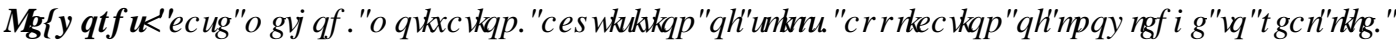
FUMFDOMHQH

\section{HXP HQ]}

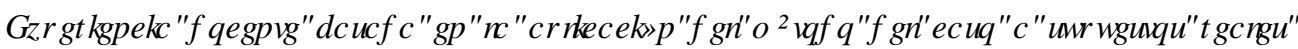

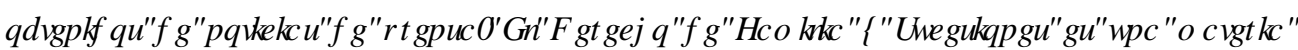

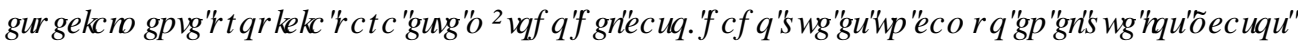

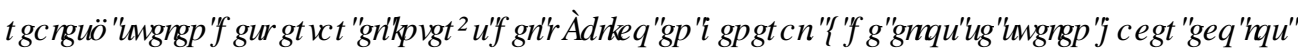

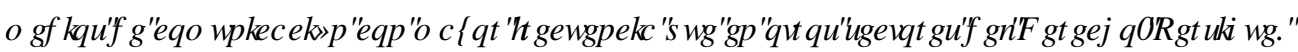

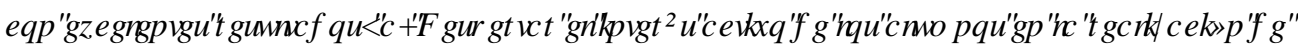

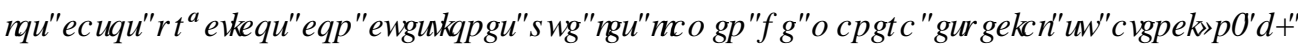

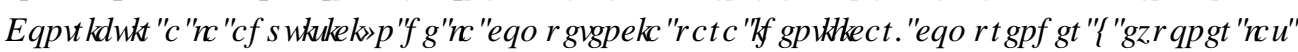

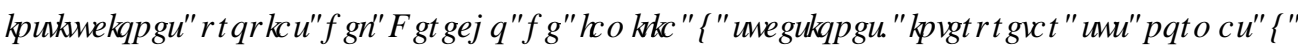

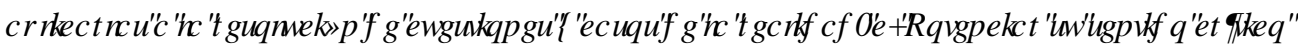
DQUA QRV P HGRV GH FRP XQIFDFIY QI SLRSRLFIRQDQCR] KHUDP IHQWV SDW GUFUP IQDU OVV

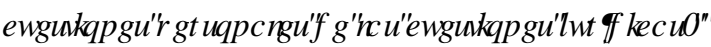

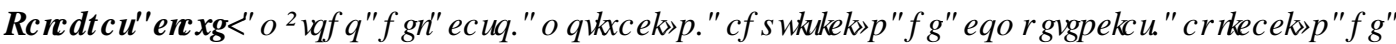

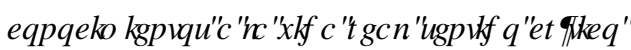




\section{Introducción}

\subsection{Contexto académico de la experiencia}

El contexto académico en el que se ha producido la intervención educativa ha sido la asignatura Derecho civil IV. La experiencia se inició en el marco del Proyecto "Preparación y sistematización de materiales docentes de Derecho de Familia en el marco del EEES (ICE-Universidad de Zaragoza, IP Sofía de Salas Murillo, PIIDUZ 09-2-172)" y se ha seguido utilizando con éxito, constatado en las encuestas realizadas por los alumnos

\subsection{Coordenadas que han servido como punto de partida}

Se han tenido como coordenadas dos puntos de partida

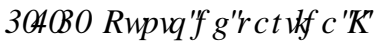

El estudiante quiere realizar las actividades propuestas en la parte práctica de la asignatura con el doble objetivo de:

a) superar los créditos correspondientes

b) facilitar la comprensión y aprendizaje de los contenidos teóricos

Pero normalmente no se constata un interés real o personal por los contenidos de estas actividades.

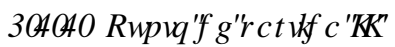

En general, el estudiante joven no suele leer la prensa tradicional escrita salvo

a) cuando se proporciona gratuitamente algunos días en la Facultad ( $O B D V$, / DD5 DlyQ

b) prensa gratuita: $4 X p$, प्P IQXURV, etc.

c) pero sobre todo y fundamentalmente, noticias que aparecen como destacadas cuando utilizan algún buscador como yahoo.

En las redes sociales, especialmente Facebook y Twitter, leen algunas noticias relacionadas con hechos curiosos, personajes famosos, o cuestiones de actualidad convertidas en WHQDQJIURSIF.

Con este método, se trata de aprovechar un campo en el que el profesor puede servirse de las herramientas enumeradas en el Punto de partida II para conseguir no solo el doble interés descrito en el Punto de Partida I, sino también el tercero: un interés real o personal por los contenidos de las actividades prácticas, lo que en términos docentes denominamos "motivación", que multiplica exponencialmente el éxito de la asunción de conocimientos y el espíritu crítico. 


\section{Objetivos}

Los objetivos propuestos han sido:

1. Despertar el interés activo de los alumnos en la realización de los casos prácticos

2. Contribuir a la adquisición de la competencia para identificar, comprender y exponer las instituciones propias del Derecho de familia y sucesiones, interpretar sus normas y aplicarlas a la resolución de cuestiones y casos de la realidad

3. Potenciar su sentido crítico ante los medios de comunicación, proporcionando herramientas para traducir a términos jurídicos noticias expuestas con carácter divulgativo.

\section{Desarrollo de la innovación}

\subsection{Justificación de la elección de la asignatura para la experiencia}

La materia de Derecho de Familia y Sucesiones es especialmente propicia para el método del caso basado en noticias de prensa.

Algunos ejemplos objeto de esta experiencia docente:

1) La herencia de Rocío Jurado: los legados superaron la porción de la que podía disponer sin lesionar la legítima de sus hijos: natural y adoptivos

2) La herencia de Camilo José Cela y el litigio con su hijo Camilo Cela Conde: el "Miró apuñalado" como modo de cubrir la legítima de Cela Conde.

3) La "desheredación" de Borja Thyssen respecto a la futura herencia de su madre Carmen Cervera.

4) Algunas reclamaciones de filiación, con aspectos jurídicos especialmente relevantes:

4.a) Del caso de "El cordobés": el valor de la negativa a someterse a las pruebas de paternidad.

4.b) Del caso de la reclamación de paternidad a Ernesto Koplowitz Stenberg: paternidad reconocida 50 años después del fallecimiento del padre: aspectos personales y sucesorios

5) Custodia del menor con progenitores separados: Kiko Rivera y Jessica Bueno: traslado de menor por vías de hecho, y solicitud de custodia compartida con progenitores residentes en distintas localidades.

6) Caso Belén Esteban y su hija Andrea: denuncia del Defensor del menor de la C.A. de Madrid y posible privación de custodia por sobreexposición de intimidad a medios de comunicación.

Por supuesto, este método se puede aplicar en cualquiera de las otras asignaturas de Derecho civil: señaladamente en Derecho de la persona (adquisición de la nacionalidad por deportistas de élite, litigios por vulneración del derecho a la intimidad y el honor de personajes públicos, etc.), pero también en Derecho de obligaciones y contratos (configuración de las cláusulas de rescisión en los contratos de los futbolistas), y, como indicamos, en general en la disciplina de Derecho civil. 


\subsection{Metodología}

Se han desarrollado dos tipos de actividades:

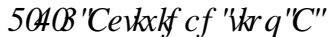

- Se proporciona el caso en forma de noticia de un periódico (haciendo referencia a su fuente) y el alumno debe:

1) Identificar cuál o cuáles son los problemas jurídicos que se plantean.

2) Buscar las normas jurídicas aplicables.

3) Identificar si hay incorrecciones o imprecisiones jurídicas en el modo de redactar la noticia.

4) Proponer una solución jurídica adecuada a Derecho, con los condicionamientos de que la noticia no suele contener todos los datos.

5) Si se ha dictado sentencia, buscarla e interpretarla (las sentencias que se reflejan en las noticias no siempre incorporan la fecha y ahí han de realizar labores de búsqueda).

- Se citan cinco ejemplos (pueden verse otros en el libro publicado, citado al final del trabajo) que se han demostrado positivos y han suscitado el interés de los alumnos, aplicando acertadamente las normas jurídicas necesarias:

I. Lea la siguiente noticia de prensa:

http://quemedices.diezminutos.es/noticias-famosos/tita-deshereda-a-su-hijo-borja

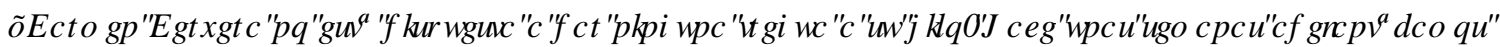

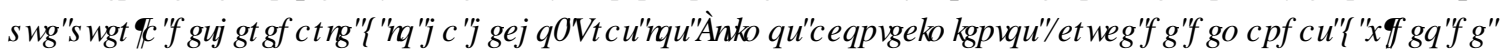

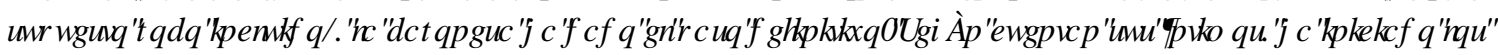

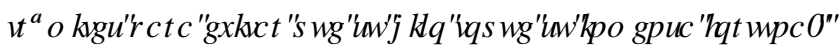

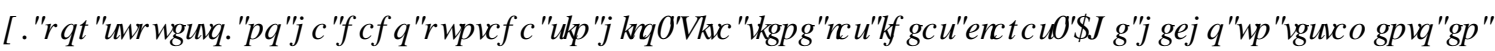

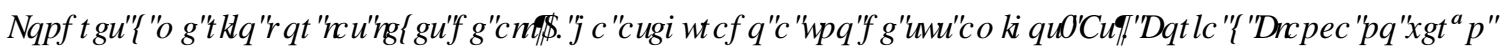
QWXQHXLR' س

Sobre esta noticia, el alumno ha de:

1) Identificar cuál o cuáles son los problemas jurídicos que se plantean ¿Qué significa desheredar? ¿Los hijos tienen derecho a la herencia o a una parte de ella? ¿Concurre causa de desheredación?

2) Buscar la norma jurídica aplicable

¿Qué legislación se aplica: Código civil o legislaciones forales? ¿Puede hacer testamento en Londres? ¿A qué legislación se sujetaría? ¿qué valor tendría ese testamento en España?

3) Identificar si hay incorrecciones o imprecisiones jurídicas en el modo de redactar la noticia ¿Qué sentido tiene nombrar que la nuera "no verá un euro" si no es legitimaria?

- Relación entre el régimen económico matrimonial y las herencias adquiridas por uno de los cónyuges.

- En otra noticia referida a este mismo tema, se dice que "Según ha hecho saber a la prensa, el testamento es inexpugnable y ni su vástago ni su nuera percibirán nada una vez que la baronesa fallezca". ¿Es correcto jurídicamente decir que un testamento es "inexpugnable”? 
4) Proponer una solución jurídica adecuada a Derecho, con los condicionamientos de que la noticia no suele contener todos los datos. Respeto a la legítima estricta del hijo.

5) Si se ha dictado sentencia, buscarla e interpretarla (las sentencias que se reflejan en las noticias no siempre incorporan la fecha y ahí han de realizar labores de búsqueda).

Respecto a las demandas citadas,

www.europapress.es/.../noticia-carmen-cervera-habla-juicio-contraborja...

Respecto a la hipotética desheredación: ¿cabe demanda mientras no haya fallecido la testadora?

II. Busque ejemplos de cómputo del grado de parentesco entre personajes históricos o famosos: cuál es el grado y porqué. Por ejemplo, el parentesco que ligaba entre sí a los Reyes católicos, o el que liga a los mellizos de Enrique Iglesias y Anna Kournikova con las hijas gemelas de Julio Iglesias y Miranda. Puede escoger otros ejemplos y exponerlos en clase.

III. Califique la validez jurídica en España — fundamentando su respuesta positiva o negativa - de estos casos de matrimonio que han aparecido en la prensa:

1) La hija de la cantante Whitney Houston, Bobbi Kristina, se casa con Nick Gordon, su hermano adoptivo

http://www.abc.es/estilo/gente/20140113/abci-boda-hija-whitney-houston-201401131125.html

2) Woody Allen se casó en 1997 con la hija adoptiva de la que hasta entonces era su mujer (Mía Farrow), que ella había adoptado con su anterior marido.

http://peru.com/entretenimiento/cine/woody-allen-soon-yi-hijastra-que-hoy-su-esposa-fotosnoticia227168

3) Una mujer se casa con su perra.

http://televicentro.hn/nota/2014/3/12/mujer-se-divorcia-del-marido-y-se-casa-con-una-perraporque-lahace-re $\% \mathrm{C} 3 \% \mathrm{ADr}$

4) Dos hombres han firmado un acuerdo en Kenia para compartir una mujer y no pelearse por ella.

http://www.abc.es/sociedad/20130826/abci-keniatas-contrato-compartir-mujer201308261745.html

5) Una mujer se casa consigo misma tras llegar soltera a los 406.

http://www.abc.es/sociedad/20150131/abci-boda-sola-eeuu-201501311237.html

IV. En la siguiente noticia de prensa se informa de que un juzgado obliga a la cantante Chenoa a hacerse una prueba biológica.

1) ¿De qué tipo de acción de filiación se trata? Aluda al artículo o artículos concretos del Código civil donde encontraría acomodo

2) ¿Tiene obligación de someterse a ella?

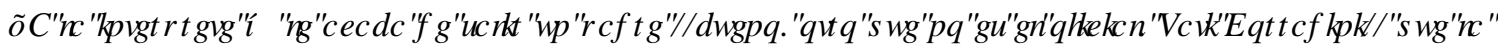

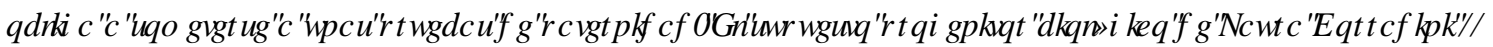

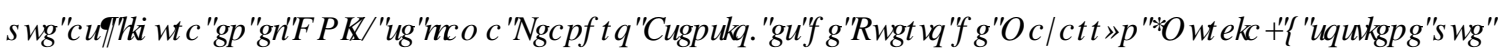

(c)) BY-NC-ND 2021, Universitat Politècnica de València

CRQJUMR, Q15 HFापिए। 


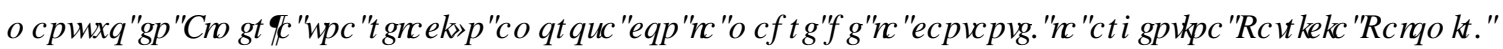
FXDQRRIFRUTDHOYHDQRIGHITITI

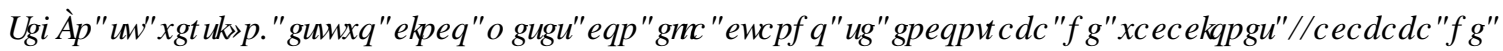

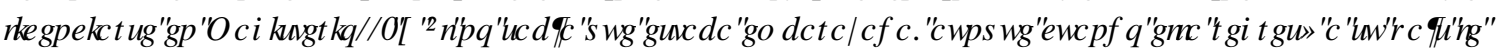

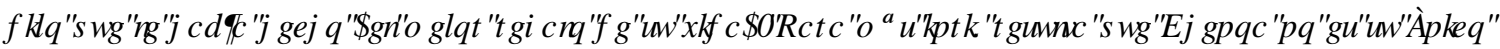

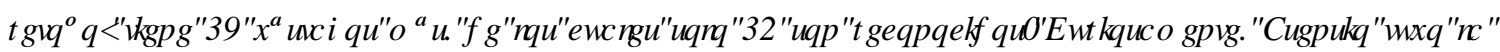

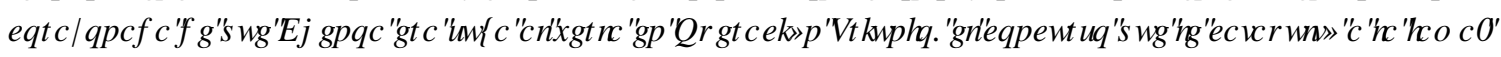

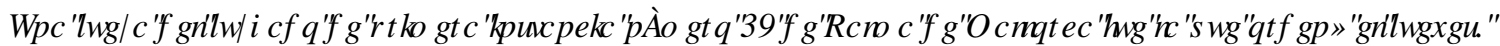

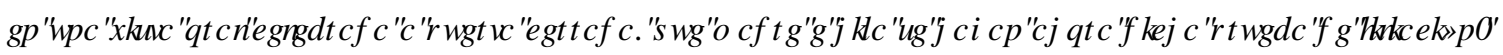

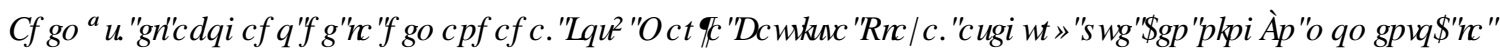

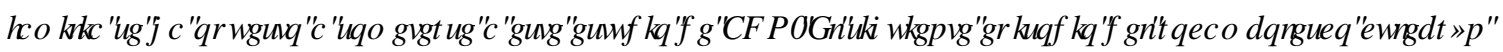

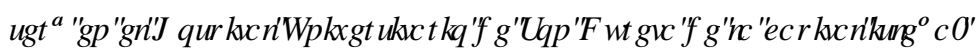

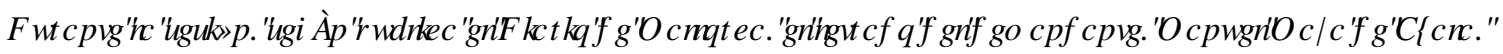

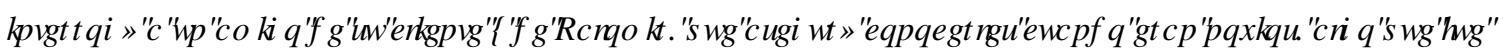

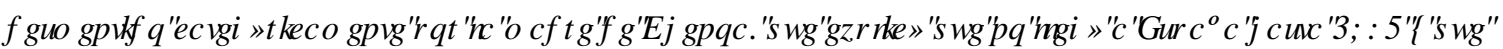

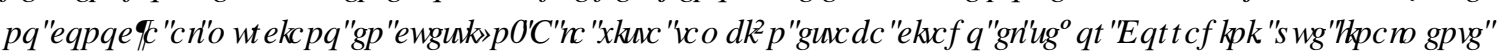

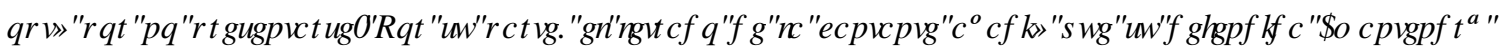

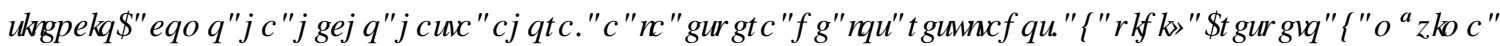

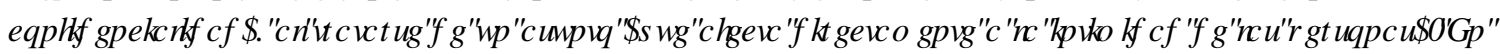

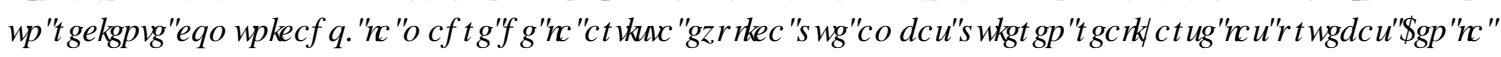

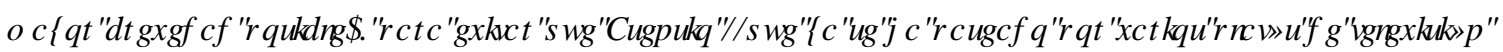

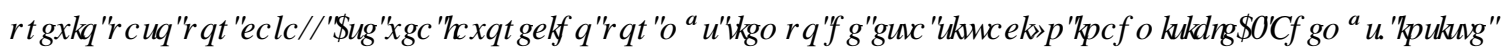

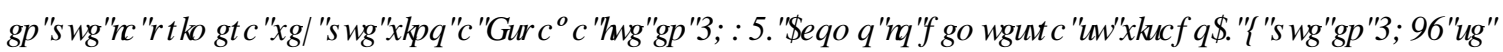

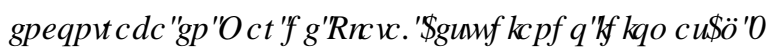

V. Busque información actualizada respecto a las siguientes acciones de filiación, detallando qué tipo de acción piensa que se habrá entablado en cada caso, y sus consecuencias en materia de alimentos, apellidos y derechos hereditarios:

1) Reclamación interpuesta por Carlos Iglesias Rangel para la declaración de su filiación respecto al rico industrial Ernst Koplowitz Steinberg, fallecido en 1962.

2) Reclamación interpuesta por José Daniel Arellán para su declaración de filiación respecto al cantante Carlos Baute, y en especial, respecto a su reclamación de 900 euros al mes durante cinco años en concepto de pensión de alimentos y de 90.000 euros en concepto de indemnización por daños y perjuicios.

3) Reclamación interpuesta por Manuel Díaz "El Cordobés” para su declaración de filiación respecto

[

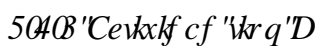

Se pide al alumno que busque noticias relativas a un determinado tema o figura que se haya explicado en clase a lo largo del curso y realice las actividades descritas dándoles forma de dictamen razonado.

La experiencia ha resultado positiva y los alumnos han demostrado interés respecto a lo relacionado con las invasiones en los derechos constitucionales al honor, la propia imagen o la intimidad, protegidos en 
España por la Constitución (desarrollada en este punto por la LO 1/1982): han sabido buscar noticias y diversos pronunciamientos jurisprudenciales, organizando la clase en distintos grupos, y exponiendo cada uno de ellos un caso, generalmente referido a personajes de todos conocidos, lo que como se indica, ha despertado su interés y hecho que vieran parte de la "utilidad" de la asignatura que estudian. La experiencia se completa con la exposición en forma de LROSQD IQJ en la que un alumno ejerce de demandante (víctima de la invasión en la privacidad, por ej.), otro de demandado (empresa propietaria del medio de comunicación) y otro de juez.

\section{Resultados}

1. Incremento de la participación en el aula:

Frente al nivel de participación en clases prácticas tradicionales (casos prácticos de laboratorio o extraídos de sentencias), que suele rondar el $20 \%$ de los presentes en el aula, la participación observada con el método de noticias de prensa se dispara al $50 \%$ de los presentes con las actividades de tipo A y al $70 \%$ con las actividades de tipo B, sobre todo las que implican la división de la clase en grupos y las de exposición en forma de de UROSQ LQJ]

2. Mejora del análisis jurídico realizado y de la adquisición de conocimientos:

Mientras que la valoración media del análisis jurídico realizado en las prácticas tradicionales es de 6 sobre 10, la valoración media del análisis en las prácticas del método de noticias de prensa es de 8'75 sobre 10. Lo cual es un indicio excelente de adquisición de conocimientos, sobre todo, si se tiene en cuenta que no se trata de la nota final a la que el alumno llega después de recorrer el curso, de superar los distintos hitos y del correspondiente estudio final de la materia, sino que es la valoración del trabajo realizado, precisamente, durante el recorrido, cuando todavía no se han completado todas las etapas. Esta valoración se extrae de las anotaciones que el profesor hace en cada clase sobre las distintas intervenciones de los alumnos y grupos, así como de las pruebas evaluables intermedias.

3. Lo anterior implica que el método, sin buscarlo, se ha revelado como una interesante herramienta de ICSSHGFF(DWRRP o clase invertida, con los beneficios de sobra conocidos que esta conlleva. Se ha observado que la búsqueda y lectura de noticias bajo la óptica del Derecho civil, y en especial del Derecho de familia, propicia que los alumnos se adelanten al estudio de materias que todavía no han sido explicadas en clase con el fin de comprender el porqué de la noticia o de buscar soluciones. Ello sin duda contribuye a un mayor aprovechamiento de la clase teórica que se traduce en un mejor nivel de comprensión y de retención de conocimientos. No es difícil para el profesor percibir esto a la vista de: i) la calidad de las intervenciones (preguntas formuladas por los propios estudiantes y respuestas a los retos lanzados por el docente) en las clases teóricas con el método expuesto, notablemente superior a la calidad de las intervenciones habituales con los métodos clásicos; ii) de las calificaciones finales.

4. Aumento de la motivación y mejora de la actitud de los estudiantes hacia sus estudios universitarios, en concreto, hacia la asignatura.

Las conductas expuestas en los números anteriores son indicios del aumento de la motivación. Esta mejora de la actitud se aprecia también en: i) las tutorías personales con los alumnos tanto desde el punto de vista sustantivo (mejoran en profundidad) como desde una perspectiva cuantitativa (se incrementan en un $20 \%$ ); en ellas es frecuente además que los propios estudiantes pongan de relieve esta circunstancia de modo explícito; ii) y en las encuentas.

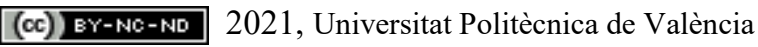

CRQJ UHRT, Q5 HGHपिए। 
5. Repercursión positiva en las calificaciones finales de la asignatura tomando como referencia calificaciones obtenidas en cursos con impartición de la docencia al modo tradicional en Derecho.

Lo expuesto en los puntos anteriores se traduce finalmente en una mejora de las calificaciones de la asignatura. Tomando como referencia las calificaciones finales de 5 grupos con método tradicional y de 5 grupos con método de noticias de prensa, se observa que las segundas son un $30 \%$ mejores que las primeras.

6. Valoración positiva por el alumnado de estas herramientas:

- Como ejemplos útiles que les facilitan el aprendizaje de los contenidos teóricos.

- Como estímulo para el aprendizaje al ver la conexión de las disciplinas académicas con la realidad.

- Como incentivo para el desarrollo de su espíritu crítico

- Como factor de habilidad social, para poder compartir estos conocimientos con personas ajenas a esta disciplina.

- Como elemento integrador en la sociedad

Esta valoración se obtiene de dos fuentes: i) las encuentas oficiales de la universidad sobre el desarrollo de la docencia del grupo; ii) las encuentas preparadas $D G K R E$ por los profesores y respondidas en clase voluntariamente por los alumnos. Los puntos destacados en el listado son el compendio del apartado de libre desarrollo que figura en la segunda modalidad de encuentas de los últimos cursos con el método.

7. Identificación por el docente de los focos de atención de un importante sector de alumnos: este tipo de noticias suele ser conocida con mayor o menor detalle y precisión jurídica por la casi totalidad de los estudiantes, a diferencia de otros temas, incluso aunque estos sean de interés general.

Se trata de aplicar dos de los factores del análisis DAFO para convertir esta "debilidad" en una "oportunidad".

8. Apreciación de indicadores de género: dependiendo de la materia, se ha observado un mayor interés e iniciativa: por ejemplo, en los casos en los que se han utilizado noticias de deportistas o relacionadas con la nacionalidad se aprecia una notable mayoría de intervención masculina, incluso en alumnos que habitualmente no participan.

\section{Conclusiones}

El método del caso basado en noticias de prensa se presenta como herramienta docente innovadora respecto del método tradicional de prácticas usado en las ciencias jurídicas (a partir de casos de laboratorio o extraídos de sentencias).

Aprovecha la información que el estudiante universitario medio recibe a través de prensa gratuita, buscadores de Internet y redes sociales para construir clases a partir de casos reales de personas de interés público.

La metología utilizada para dicha construcción se descompone en dos tipos de actividades con distinta implicación del alumno: i) La entrega de casos ya extraídos de la prensa por el docente y la fijación de la tarea guiada que el alumno debe realizar (identificación de problemas jurídicos, búsqueda de normas aplicables, identificación de imprecisiones o incorrecciones técnicas en la noticia, propuesta de solución 
adecuada a Derecho, búsqueda e interpretación-análisis de la sentencia que resuelve el caso). ii) La búsqueda por el propio estudiante de las noticias sobre las que trabajar y la exposición del análisis en grupos o en forma de UROSQI IQJ. Aunque este segundo tipo de actividad exige un esfuerzo extra de identificación, selección y elaboración propia, para lo cual precisa de mayor preparación y estudio, los resultados son ligeramente- superiores a los obtenidos con la primera actividad más básica.

La innovación del método produce resultados satisfactorios que mejora de forma general la enseñanza de la asignatura. En particular, hay que destacar que el incremento de la participación en el aula práctica, la mejora significativa del análisis jurídico objetivamente cuantificada en la docencia práctica, la mejor calidad de las intervenciones en las clases teóricas, la propiciación del[PRGXVRSHDQALde IOSSHCFPOWRRP con sus beneficios, el aumento del número y nivel de profundidad técnico-jurídico de las tutorías, la propia valoración del estudiantado y el dato objetivo e incontestable de la mejora de resultados académicos son indicios de que el método del caso de las noticias de prensa facilita en mayor medida que el método clásico: i) La transmisión de conocimientos en Derecho: el alumno obtiene beneficios desde un punto de vista sustantivo. ii) El desarrollo de competencias hermenéuticas y de aplicación del ordenamiento jurídico al caso concreto: el estudiante obtiene beneficios técnicos y de procedimiento jurídico, especialmente útiles para el ejercicio de las profesiones jurídicas. iii) El despertar de la motivación y del espíritu crítico: el alumnado obtiene beneficios actitudinales personales. iv) La integración en la sociedad circundante mediante la conciencia de la utilidad real de sus conocimientos universitarios: el estudiantado obtiene beneficios sociales. La mejora en las cuatro dimensiones básicas de la docencia en Derecho (sustantiva, técnico-procedimental, actitudinal y social) resulta concluyente como método de innovación útil y recomendable.

\section{Referencias}

Algunos de los casos y materiales a utilizar han sido publicados en:

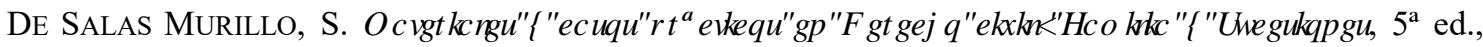
Zaragoza, Ed. Kronos, 2018, ISBN 9788496267329 